\title{
Measurement of the Triple Gluon Vertex from double quark tagged 4-Jet Events
}

DELPHI Collaboration

\begin{abstract}
The 4-jet events collected by the DELPHI experiment at LEP1 in 1992, 1993 and 1994 are analysed to determine the contribution of the triple-gluon vertex. Two of the four jets are tagged as jets from $b$ - or $c$-quarks using lifetime and lepton transverse momentum information. The 4-jet contributions from doublegluon bremsstrahlung, the triple-gluon vertex, and secondary quark-antiquark pair production then yield significantly different two-dimensional distributions in the generalized Nachtmann Reiter angle versus the opening angle of the two secondary jets. These distributions are used to fit $C_{A} / C_{F}$, the ratio of the coupling strength of the triple-gluon vertex to that of gluon bremsstrahlung, and $N_{C} / N_{A}$, the ratio of the number of quark colours to the number of gluons, giving

$$
C_{A} / C_{F}=2.51 \pm 0.28 \text { and } N_{C} / N_{A}=0.38 \pm 0.10
$$
\end{abstract}

in agreement with the values expected from QCD:

$$
C_{A} / C_{F}=9 / 4=2.25 \text { and } N_{C} / N_{A}=3 / 8=0.375 \text {. }
$$


P.Abreu ${ }^{21}$, W.Adam ${ }^{49}$, T.Adye ${ }^{36}$, P.Adzic ${ }^{11}$, G.D.Alekseev ${ }^{16}$, R.Alemany ${ }^{48}$, P.P.Allport ${ }^{22}$, S.Almehed $^{24}$, U.Amaldi $^{9}$, S.Amato ${ }^{46}$, P.Andersson ${ }^{43}$, A.Andreazza ${ }^{9}$, P.Antilogus ${ }^{9}$, W-D.Apel ${ }^{17}$, Y.Arnoud ${ }^{14}$, B.Asman $^{43}$, J-E.Augustin ${ }^{25}$, A.Augustinus ${ }^{30}$, P.Baillon ${ }^{9}$, P.Bambade ${ }^{19}$, F.Barao ${ }^{21}$, M.Barbi ${ }^{46}$, G.Barbiellini ${ }^{45}$, D.Y.Bardin ${ }^{16}$, G.Barker ${ }^{9}$, A.Baroncelli ${ }^{39}$, O.Barring ${ }^{24}$, M.J.Bates ${ }^{36}$, M.Battaglia $^{15}$, M.Baubillier $^{23}$, J.Baudot $^{38}$, K-H.Becks ${ }^{51}$, M.Begalli ${ }^{6}$, P.Beilliere ${ }^{8}$, Yu.Belokopytov ${ }^{9,52}$, A.C.Benvenuti ${ }^{5}$, C.Berat ${ }^{14}$, M.Berggren $^{46}{ }^{4}$ D.Bertini $^{25}$, D.Bertrand ${ }^{2}$, M.Besancon ${ }^{38}$, F.Bianchi ${ }^{44}$, M.Bigi ${ }^{44}$, M.S.Bilenky ${ }^{16}$, P.Billoir ${ }^{23}$, M-A.Bizouard $^{19}$, D.Bloch $^{10}$, M.Blume ${ }^{51}$, M.Bonesini ${ }^{27}$, W.Bonivento ${ }^{27}$, M.Boonekamp ${ }^{38}$, P.S.L.Booth ${ }^{22}$, A.W.Borgland ${ }^{4}$, G.Borisov $^{38,41}$, C.Bosio ${ }^{39}$, O.Botner ${ }^{47}$, E.Boudinov ${ }^{30}$, B.Bouquet ${ }^{19}$, C.Bourdarios ${ }^{19}$, T.J.V.Bowcock ${ }^{22}$, M.Bozzo $^{13}$, P.Branchini ${ }^{39}$, K.D.Brand ${ }^{35}$, T.Brenke ${ }^{51}$, R.A.Brenner ${ }^{47}$, R.C.A.Brown ${ }^{9}$, P.Bruckman $^{18}$, J-M.Brunet $^{8}$, L.Bugge ${ }^{32}$, T.Buran ${ }^{32}$, T.Burgsmueller ${ }^{51}$, P.Buschmann ${ }^{51}$, S.Cabrera ${ }^{48}$, M.Caccia ${ }^{27}$, M.Calvi ${ }^{27}$, A.J.Camacho Rozas ${ }^{40}$, T.Camporesi ${ }^{9}$, V.Canale ${ }^{37}$, M.Canepa ${ }^{13}$, F.Carena ${ }^{9}$, L.Carroll ${ }^{22}$, C.Caso $^{13}$, M.V.Castillo Gimenez ${ }^{48}$, A.Cattai ${ }^{9}$, F.R.Cavallo ${ }^{5}$, V.Chabaud ${ }^{9}$, Ph.Charpentier ${ }^{9}$, L.Chaussard $^{25}$, P.Checchia $^{35}$, G.A.Chelkov ${ }^{16}$, M.Chen ${ }^{2}$, R.Chierici ${ }^{44}$, P.Chliapnikov ${ }^{41}$, P.Chochula ${ }^{7}$, V.Chorowicz ${ }^{25}$, J.Chudoba ${ }^{29}$ V.Cindro ${ }^{42}$, P.Collins ${ }^{9}$, M.Colomer ${ }^{48}$, R.Contri ${ }^{13}$, E.Cortina ${ }^{48}$, G.Cosme ${ }^{19}$, F.Cossutti ${ }^{45}$, J-H.Cowell ${ }^{22}$, H.B.Crawley ${ }^{1}$, D.Crennell ${ }^{36}$, G.Crosetti ${ }^{13}$, J.Cuevas Maestro ${ }^{33}$, S.Czellar ${ }^{15}$, J.Dahm ${ }^{51}$, B.Dalmagne ${ }^{19}$, G.Damgaard $^{28}$, P.D.Dauncey ${ }^{36}$, M.Davenport ${ }^{9}$, W.Da Silva ${ }^{23}$, A.Deghorain ${ }^{2}$, G.Della Ricca ${ }^{45}$, P.Delpierre ${ }^{26}$, N.Demaria $^{34}$, A.De Angelis ${ }^{9}$, W.De Boer ${ }^{17}$, S.De Brabandere ${ }^{2}$, C.De Clercq ${ }^{2}$, C.De La Vaissiere ${ }^{23}$, B.De Lotto ${ }^{45}$, A.De Min ${ }^{35}$, L.De Paula ${ }^{46}$, H.Dijkstra ${ }^{9}$, L.Di Ciaccio ${ }^{37}$, A.Di Diodato ${ }^{37}$, A.Djannati $^{8}$, J.Dolbeau $^{8}$, K.Doroba ${ }^{50}$, M.Dracos $^{10}$, J.Drees ${ }^{51}$, K.-A.Drees ${ }^{51}$, M.Dris ${ }^{31}$, J-D.Durand ${ }^{25,9}$, D.Edsall ${ }^{1}$, R.Ehret ${ }^{17}$, G.Eigen ${ }^{4}$, T.Ekelof ${ }^{47}$, G.Ekspong ${ }^{43}$, M.Elsing ${ }^{9}$, J-P.Engel ${ }^{10}$, B.Erzen ${ }^{42}$, M.Espirito Santo ${ }^{21}$, E.Falk ${ }^{24}$, G.Fanourakis $^{11}$, D.Fassouliotis ${ }^{45}$, M.Feindt ${ }^{9}$, A.Fenyuk ${ }^{41}$, P.Ferrari ${ }^{27}$, A.Ferrer ${ }^{48}$, S.Fichet ${ }^{23}$, T.A.Filippas ${ }^{31}$, A.Firestone $^{1}$, P.-A.Fischer ${ }^{10}$, H.Foeth ${ }^{9}$, E.Fokitis ${ }^{31}$, F.Fontanelli ${ }^{13}$, F.Formenti ${ }^{9}$, B.Franek ${ }^{36}$, A.G.Frodesen ${ }^{4}$, R.Fruhwirth $^{49}$, F.Fulda-Quenzer ${ }^{19}$, J.Fuster ${ }^{48}$, A.Galloni ${ }^{22}$, D.Gamba ${ }^{44}$, M.Gandelman ${ }^{46}$, C.Garcia $^{48}$, J.Garcia $^{40}$, C.Gaspar ${ }^{9}$, U.Gasparini ${ }^{35}$, Ph.Gavillet ${ }^{9}$, E.N.Gazis ${ }^{31}$, D.Gele ${ }^{10}$, J-P.Gerber ${ }^{10}$, L.Geryukov $^{41}$, R.Gokieli ${ }^{50}$, B.Golob ${ }^{42}$, P.Goncalves ${ }^{21}$, G.Gopal $^{36}$, L.Gorn ${ }^{1}$, M.Gorski $^{50}$, Yu.Gouz ${ }^{44,52}$, V.Gracco ${ }^{13}$,

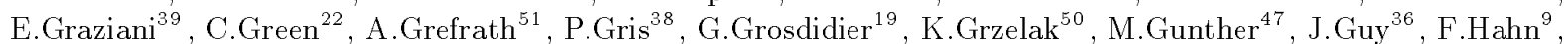
S.Hahn $^{51}$, Z.Hajduk ${ }^{18}$, A.Hallgren ${ }^{47}$, K.Hamacher ${ }^{51}$, F.J.Harris ${ }^{34}$, V.Hedberg ${ }^{24}$, R.Henriques ${ }^{21}$, J.J.Hernandez $^{48}$, P.Herquet ${ }^{2}$, H.Herr ${ }^{9}$, T.L.Hessing ${ }^{34}$, J.-M.Heuser ${ }^{51}$, E.Higon ${ }^{48}$, S-O.Holmgren $^{43}$, P.J.Holt $^{34}$, D.Holthuizen $^{30}$, S.Hoorelbeke ${ }^{2}$, M.Houlden ${ }^{22}$, J.Hrubec $^{49}$, K.Huet ${ }^{2}$, K.Hultqvist ${ }^{43}$, J.N.Jackson ${ }^{22}$, R.Jacobsson ${ }^{43}$, P.Jalocha ${ }^{9}$, R.Janik ${ }^{7}$, Ch.Jarlskog ${ }^{24}$, G.Jarlskog ${ }^{24}$, P.Jarry ${ }^{38}$, B.Jean-Marie ${ }^{19}$, E.K.Johansson ${ }^{43}$, L.Jonsson $^{24}$, P.Jonsson ${ }^{24}$, C.Joram ${ }^{9}$, P.Juillot ${ }^{10}$, M.Kaiser ${ }^{17}$, F.Kapusta ${ }^{23}$, K.Karafasoulis ${ }^{11}$, E.Karvelas ${ }^{11}$, S.Katsanevas $^{25}$, E.C.Katsoufis ${ }^{31}$, R.Keranen ${ }^{4}$, Yu.Khokhlov ${ }^{41}$, B.A.Khomenko ${ }^{16}$, N.N.Khovanski ${ }^{16}$, B.King ${ }^{22}$, N.J.Kjaer ${ }^{30}$, O.Klapp ${ }^{51}$, H.Klein ${ }^{9}$, P.Kluit ${ }^{30}$, D.Knoblauch ${ }^{17}$, P.Kokkinias ${ }^{11}$, A.Konopliannikov ${ }^{41}$, M.Koratzinos $^{9}$, K.Korcyl ${ }^{18}$, V.Kostioukhine ${ }^{41}$, C.Kourkoumelis ${ }^{3}$, O.Kouznetsov ${ }^{16}$, C.Kreuter ${ }^{9}$, I.Kronkvist ${ }^{24}$, Z.Krumstein $^{16}$, W.Krupinski ${ }^{18}$, P.Kubinec ${ }^{7}$, W.Kucewicz ${ }^{18}$, K.Kurvinen ${ }^{15}$, C.Lacasta ${ }^{9}$, I.Laktineh ${ }^{25}$, J.W.Lamsa ${ }^{1}$, L.Lanceri ${ }^{45}$, D.W.Lane ${ }^{1}$, P.Langefeld ${ }^{51}$, J-P.Laugier ${ }^{38}$, R.Lauhakangas ${ }^{15}$, G.Leder $^{49}$, F.Ledroit $^{14}$, V.Lefebure $^{2}$, C.K.Legan ${ }^{1}$, A.Leisos ${ }^{11}$, R.Leitner ${ }^{29}$, J.Lemonne ${ }^{2}$, G.Lenzen ${ }^{51}$, V.Lepeltier ${ }^{19}$, T.Lesiak $^{18}{ }^{\text {, }}$, M.Lethuillier $^{38}$, J.Libby ${ }^{34}$, D.Liko ${ }^{9}$, A.Lipniacka ${ }^{43}$, I.Lippi ${ }^{35}$, B.Loerstad ${ }^{24}$, J.G.Loken ${ }^{34}$, J.M.Lopez ${ }^{40}$, D.Loukas ${ }^{11}$, P.Lutz ${ }^{38}$, L.Lyons ${ }^{34}$, J.MacNaughton ${ }^{49}$, G.Maehlum ${ }^{17}$, J.R.Mahon ${ }^{6}$, A.Maio ${ }^{21}$, T.G.M.Malmgren ${ }^{43}$, V.Malychev ${ }^{16}$, F.Mandl ${ }^{49}$, J.Marco ${ }^{40}$, R.Marco ${ }^{40}$, B.Marechal ${ }^{46}$, M.Margoni $^{35}$, J-C.Marin $^{9}$, C.Mariotti ${ }^{9}$, A.Markou ${ }^{11}$, C.Martinez-Rivero ${ }^{33}$, F.Martinez-Vidal ${ }^{48}$, S.Marti i Garcia ${ }^{22}$, J.Masik ${ }^{29}$, F.Matorras $^{40}$, C.Matteuzzi ${ }^{27}$, G.Matthiae ${ }^{37}$, M.Mazzucato ${ }^{35}$, M.Mc Cubbin ${ }^{22}$, R.Mc Kay ${ }^{1}$, R.Mc Nulty ${ }^{9}$, G.Mc Pherson $^{22}$, J.Medbo ${ }^{47}$, C.Meroni ${ }^{27}$, S.Meyer ${ }^{17}$, W.T.Meyer ${ }^{1}$, A.Miagkov ${ }^{41}$, M.Michelotto ${ }^{35}$, E.Migliore ${ }^{44}$, L.Mirabito $^{25}$, W.A.Mitaroff ${ }^{49}$, U.Mjoernmark ${ }^{24}$, T.Moa ${ }^{43}$, R.Moeller ${ }^{28}$, K.Moenig ${ }^{9}$, M.R.Monge ${ }^{13}$, P.Morettini $^{13}$, H.Mueller ${ }^{17}$, K.Muenich ${ }^{51}$, M.Mulders ${ }^{30}$, L.M.Mundim ${ }^{6}$, W.J.Murray ${ }^{36}$, B.Muryn $^{14,18}$, G.Myatt $^{34}$, T.Myklebust ${ }^{32}$, F.Naraghi ${ }^{14}$, F.L.Navarria ${ }^{5}$, S.Navas ${ }^{48}$, K.Nawrocki ${ }^{50}$, P.Negri ${ }^{27}$, W.Neumann ${ }^{51}$, N.Neumeister ${ }^{49}$, R.Nicolaidou ${ }^{3}$, B.S.Nielsen ${ }^{28}$, M.Nieuwenhuizen ${ }^{30}$, V.Nikolaenko ${ }^{10}$, M.Nikolenko ${ }^{10,16}$, P.Niss ${ }^{43}$, A.Nomerotski ${ }^{35}$, A.Normand ${ }^{22}$, A.Nygren ${ }^{24}$, W.Oberschulte-Beckmann ${ }^{17}$, V.Obraztsov ${ }^{41}$, A.G.Olshevski ${ }^{16}$, A.Onofre $^{21}$, R.Orava ${ }^{15}$, G.Orazi ${ }^{10}$, S.Ortumo ${ }^{48}$, K.Osterberg ${ }^{15}$, A.Ouraou ${ }^{38}$, P.Paganini ${ }^{19}$, M.Paganoni ${ }^{9,27}$, S.Paiano $^{5}$, R.Pain ${ }^{23}$, H.Palka ${ }^{18}$, Th.D.Papadopoulou ${ }^{31}$, K.Papageorgiou ${ }^{11}$, L.Pape ${ }^{9}$, C.Parkes ${ }^{34}$, F.Parodi ${ }^{13}$, U.Parzefall $^{22}$, A.Passeri ${ }^{39}$, M.Pegoraro ${ }^{35}$, L.Peralta ${ }^{21}$, H.Pernegger ${ }^{49}$, M.Pernicka ${ }^{49}$, A.Perrotta ${ }^{5}$, C.Petridou $^{45}$, A.Petrolini $^{13}$, H.T.Phillips ${ }^{36}$, G.Piana ${ }^{13}$, F.Pierre ${ }^{38}$, E.Piotto ${ }^{35}$, T.Podobnik ${ }^{34}$, O.Podobrin ${ }^{9}$, M.E.Pol ${ }^{6}$, G.Polok $^{18}$, P.Poropat ${ }^{45}$, V.Pozdniakov ${ }^{16}$, P.Privitera ${ }^{37}$, N.Pukhaeva ${ }^{16}$, A.Pullia ${ }^{27}$, D.Radojicic ${ }^{34}$, S.Ragazzi ${ }^{27}$, H.Rahmani ${ }^{31}$, J.Rames ${ }^{12}$, P.N.Ratoff ${ }^{20}$, A.L.Read ${ }^{32}$, M.Reale ${ }^{51}$, P.Rebechi ${ }^{9}$, N.G.Redaelli ${ }^{27}$, M.Regler $^{49}$, D.Reid ${ }^{9}$, R.Reinhardt ${ }^{51}$, P.B.Renton ${ }^{34}$, L.K.Resvanis ${ }^{3}$, F.Richard ${ }^{19}$, J.Ridky ${ }^{12}$, G.Rinaudo ${ }^{44}$, O.Rohne ${ }^{32}$, A.Romero $^{44}$, P.Ronchese ${ }^{35}$, L.Roos ${ }^{23}$, E.I.Rosenberg ${ }^{1}, \quad$ P.Rosinsky ${ }^{7}, \quad$ P.Roudeau $^{19}$, T.Rovelli ${ }^{5}$, V.Ruhlmann-Kleider ${ }^{38}$, A.Ruiz ${ }^{40}$, K.Rybicki ${ }^{18}$, H.Saarikko ${ }^{15}$, Y.Sacquin ${ }^{38}$, A.Sadovsky ${ }^{16}$, G.Sajot $^{14}$, J.Salt ${ }^{48}$, M.Sannino $^{13}$, H.Schneider ${ }^{17}$, U.Schwickerath ${ }^{17}$, M.A.E.Schyns ${ }^{51}$, G.Sciolla ${ }^{44}$, F.Scuri ${ }^{45}$, P.Seager ${ }^{20}$, Y.Sedykh ${ }^{16}$, A.M.Segar ${ }^{34}$, A.Seitz ${ }^{17}$, R.Sekulin ${ }^{36}$, L.Serbelloni ${ }^{37}$, R.C.Shellard ${ }^{6}$, A.Sheridan ${ }^{22}$, P.Siegrist ${ }^{9,38}$, R.Silvestre ${ }^{38}$, F.Simonetto ${ }^{35}$, A.N.Sisakian ${ }^{16}$, T.B.Skaali ${ }^{32}$, G.Smadja ${ }^{25}$, N.Smirnov ${ }^{41}$, O.Smirnova $^{24}$, G.R.Smith $^{36}$, A.Sokolov ${ }^{41}$, O.Solovianov ${ }^{41}$, R.Sosnowski ${ }^{50}$, D.Souza-Santos ${ }^{6}$, T.Spassov ${ }^{21}$, E.Spiriti ${ }^{39}$, P.Sponholz ${ }^{51}$, S.Squarcia ${ }^{13}$, D.Stampfer ${ }^{9}$, C.Stanescu ${ }^{39}$, S.Stanic ${ }^{42}$, S.Stapnes ${ }^{32}$, I.Stavitski ${ }^{35}$, K.Stevenson ${ }^{34}$, A.Stocchi $^{19}$, J.Strauss ${ }^{49}$, R.Strub ${ }^{10}$, B.Stugu ${ }^{4}$, M.Szczekowski ${ }^{50}$, M.Szeptycka ${ }^{50}$, T.Tabarelli ${ }^{27}$, J.P.Tavernet ${ }^{23}$, 
E.Tcherniaev ${ }^{41}$, F.Tegenfeldt ${ }^{47}$, F.Terranova ${ }^{27}$, J.Thomas ${ }^{34}$, A.Tilquin ${ }^{26}$, J.Timmermans ${ }^{30}$, L.G.Tkatchev ${ }^{16}$, T.Todorov $^{10}$, S.Todorova ${ }^{10}$, D.Z.Toet ${ }^{30}$, A.Tomaradze ${ }^{2}$, B.Tome ${ }^{21}$, A.Tonazzo ${ }^{27}$, L.Tortora ${ }^{39}$, G.Transtromer $^{24}$, D.Treille ${ }^{9}$, G.Tristram ${ }^{8}$, A.Trombini ${ }^{19}$, C.Troncon $^{27}$, A.Tsirou ${ }^{9}$, M-L.Turluer $^{38}$, I.A.Tyapkin ${ }^{16}$, M.Tyndel $^{36}$, S.Tzamarias ${ }^{11}$, B.Ueberschaer ${ }^{51}$, O.Ullaland ${ }^{9}$, V.Uvarov ${ }^{41}$, G.Valenti ${ }^{5}$, E.Vallazza ${ }^{45}$, G.W.Van Apeldoorn ${ }^{30}$, P.Van Dam ${ }^{30}$, J.Van Eldik ${ }^{30}$, A.Van Lysebetten ${ }^{2}$, N.Vassilopoulos ${ }^{34}$, G.Vegni ${ }^{27}$, L.Ventura ${ }^{35}$, W.Venus ${ }^{36}$, F.Verbeure $^{2}$, M.Verlato ${ }^{35}$, L.S.Vertogradov ${ }^{16}$, D.Vilanova ${ }^{38}$, P.Vincent ${ }^{25}$, L.Vitale ${ }^{45}$, A.S.Vodopyanov ${ }^{16}$, V.Vrba $^{12}$, H.Wahlen ${ }^{51}$, C.Walck ${ }^{43}$, C.Weiser ${ }^{17}$, A.M.Wetherell ${ }^{9}$, D.Wicke ${ }^{51}$, J.H.Wickens ${ }^{2}$, M.Wielers ${ }^{17}$, G.R.Wilkinson ${ }^{9}$, W.S.C.Williams ${ }^{34}$, M.Winter ${ }^{10}{ }^{\prime}$, M.Witek $^{18}$, T.Wlodek ${ }^{19}$, J.Yi ${ }^{1}$, K.Yip ${ }^{34}$, O.Yushchenko ${ }^{41}$, F.Zach $^{25}$, A.Zaitsev ${ }^{41}$, A.Zalewska ${ }^{9}$, P.Zalewski ${ }^{50}$, D.Zavrtanik ${ }^{42}$, E.Zevgolatakos ${ }^{11}, \quad$ N.I.Zimin ${ }^{16}$, G.C.Zucchellis ${ }^{43}$, G.Zumerle ${ }^{35}$

\footnotetext{
${ }^{1}$ Department of Physics and Astronomy, Iowa State University, Ames IA 50011-3160, USA

${ }^{2}$ Physics Department, Univ. Instelling Antwerpen, Universiteitsplein 1, B-2610 Wilrijk, Belgium and IIHE, ULB-VUB, Pleinlaan 2, B-1050 Brussels, Belgium and Faculté des Sciences, Univ. de l'Etat Mons, Av. Maistriau 19, B-7000 Mons, Belgium

${ }^{3}$ Physics Laboratory, University of Athens, Solonos Str. 104, GR-10680 Athens, Greece ${ }^{4}$ Department of Physics, University of Bergen, Allégaten 55, N-5007 Bergen, Norway

${ }^{5}$ Dipartimento di Fisica, Università di Bologna and INFN, Via Irnerio 46, I-40126 Bologna, Italy

${ }^{6}$ Centro Brasileiro de Pesquisas Físicas, rua Xavier Sigaud 150, RJ-22290 Rio de Janeiro, Brazil and Depto. de Física, Pont. Univ. Católica, C.P. 38071 RJ-22453 Rio de Janeiro, Brazil and Inst. de Física, Univ. Estadual do Rio de Janeiro, rua São Francisco Xavier 524, Rio de Janeiro, Brazil ${ }^{7}$ Comenius University, Faculty of Mathematics and Physics, Mlynska Dolina, SK-84215 Bratislava, Slovakia ${ }^{8}$ Collège de France, Lab. de Physique Corpusculaire, IN2P3-CNRS, F-75231 Paris Cedex 05, France

${ }^{9}$ CERN, CH-1211 Geneva 23, Switzerland

${ }^{10}$ Institut de Recherches Subatomiques, IN2P3 - CNRS/ULP - BP20, F-67037 Strasbourg Cedex, France

${ }^{11}$ Institute of Nuclear Physics, N.C.S.R. Demokritos, P.O. Box 60228, GR-15310 Athens, Greece

${ }^{12}$ FZU, Inst. of Physics of the C.A.S. High Energy Physics Division, Na Slovance 2, 180 40, Praha 8, Czech Republic

${ }^{13}$ Dipartimento di Fisica, Università di Genova and INFN, Via Dodecaneso 33, I-16146 Genova, Italy

${ }^{14}$ Institut des Sciences Nucléaires, IN2P3-CNRS, Université de Grenoble 1, F-38026 Grenoble Cedex, France

${ }^{15}$ Helsinki Institute of Physics, HIP, P.O. Box 9, FIN-00014 Helsinki, Finland

${ }^{16}$ Joint Institute for Nuclear Research, Dubna, Head Post Office, P.O. Box 79, 101000 Moscow, Russian Federation

${ }^{17}$ Institut für Experimentelle Kernphysik, Universität Karlsruhe, Postfach 6980, D-76128 Karlsruhe, Germany

${ }^{18}$ Institute of Nuclear Physics and University of Mining and Metalurgy, Ul. Kawiory 26a, PL-30055 Krakow, Poland

${ }^{19}$ Université de Paris-Sud, Lab. de l'Accélérateur Linéaire, IN2P3-CNRS, Bât. 200, F-91405 Orsay Cedex, France

${ }^{20}$ School of Physics and Chemistry, University of Lancaster, Lancaster LA1 4YB, UK

${ }^{21}$ LIP, IST, FCUL - Av. Elias Garcia, 14-1 ${ }^{\circ}$, P-1000 Lisboa Codex, Portugal

${ }^{22}$ Department of Physics, University of Liverpool, P.O. Box 147, Liverpool L69 3BX, UK

${ }^{23}$ LPNHE, IN2P3-CNRS, Universités Paris VI et VII, Tour 33 (RdC), 4 place Jussieu, F-75252 Paris Cedex 05, France

${ }^{24}$ Department of Physics, University of Lund, Sölvegatan 14, S-22363 Lund, Sweden

${ }^{25}$ Université Claude Bernard de Lyon, IPNL, IN2P3-CNRS, F-69622 Villeurbanne Cedex, France

${ }^{26}$ Univ. d'Aix - Marseille II - CPP, IN2P3-CNRS, F-13288 Marseille Cedex 09, France

${ }^{27}$ Dipartimento di Fisica, Università di Milano and INFN, Via Celoria 16, I-20133 Milan, Italy

${ }^{28}$ Niels Bohr Institute, Blegdamsvej 17, DK-2100 Copenhagen 0, Denmark

${ }^{29} \mathrm{NC}$, Nuclear Centre of MFF, Charles University, Areal MFF, V Holesovickach 2, 180 00, Praha 8, Czech Republic

${ }^{30}$ NIKHEF, Postbus 41882, NL-1009 DB Amsterdam, The Netherlands

${ }^{31}$ National Technical University, Physics Department, Zografou Campus, GR-15773 Athens, Greece

${ }^{32}$ Physics Department, University of Oslo, Blindern, N-1000 Oslo 3, Norway

${ }^{33}$ Dpto. Fisica, Univ. Oviedo, Avda. Calvo Sotelo, S/N-33007 Oviedo, Spain, (CICYT-AEN96-1681)

${ }^{34}$ Department of Physics, University of Oxford, Keble Road, Oxford OX1 3RH, UK

${ }^{35}$ Dipartimento di Fisica, Università di Padova and INFN, Via Marzolo 8, I-35131 Padua, Italy

${ }^{36}$ Rutherford Appleton Laboratory, Chilton, Didcot OX11 OQX, UK

${ }^{37}$ Dipartimento di Fisica, Università di Roma II and INFN, Tor Vergata, I-00173 Rome, Italy

${ }^{38} \mathrm{CEA}$, DAPNIA/Service de Physique des Particules, CE-Saclay, F-91191 Gif-sur-Yvette Cedex, France

${ }^{39}$ Istituto Superiore di Sanità, Ist. Naz. di Fisica Nucl. (INFN), Viale Regina Elena 299, I-00161 Rome, Italy

${ }^{40}$ Instituto de Fisica de Cantabria (CSIC-UC), Avda. los Castros, S/N-39006 Santander, Spain, (CICYT-AEN96-1681)

${ }^{41}$ Inst. for High Energy Physics, Serpukov P.O. Box 35, Protvino, (Moscow Region), Russian Federation

${ }^{42}$ J. Stefan Institute, Jamova 39, SI-1000 Ljubljana, Slovenia and Department of Astroparticle Physics, School of Environmental Sciences, Kostanjeviska 16a, Nova Gorica, SI-5000 Slovenia, and Department of Physics, University of Ljubljana, SI-1000 Ljubljana, Slovenia

${ }^{43}$ Fysikum, Stockholm University, Box 6730, S-113 85 Stockholm, Sweden

${ }^{44}$ Dipartimento di Fisica Sperimentale, Università di Torino and INFN, Via P. Giuria 1, I-10125 Turin, Italy

${ }^{45}$ Dipartimento di Fisica, Università di Trieste and INFN, Via A. Valerio 2, I-34127 Trieste, Italy and Istituto di Fisica, Università di Udine, I-33100 Udine, Italy

${ }^{46}$ Univ. Federal do Rio de Janeiro, C.P. 68528 Cidade Univ., Tlha do Fundão BR-21945-970 Rio de Janeiro, Brazil

${ }^{47}$ Department of Radiation Sciences, University of Uppsala, P.O. Box 535, S-751 21 Uppsala, Sweden

${ }^{48}$ IFIC, Valencia-CSIC, and D.F.A.M.N., U. de Valencia, Avda. Dr. Moliner 50, E-46100 Burjassot (Valencia), Spain

${ }^{49}$ Institut für Hochenergiephysik, Österr. Akad. d. Wissensch., Nikolsdorfergasse 18, A-1050 Vienna, Austria

${ }^{50}$ Inst. Nuclear Studies and University of Warsaw, Ul. Hoza 69, PL-00681 Warsaw, Poland

${ }^{51}$ Fachbereich Physik, University of Wuppertal, Postfach 100 127, D-42097 Wuppertal, Germany

${ }^{52}$ On leave of absence from IHEP Serpukhov
} 


\section{Introduction}

An essential feature of Quantum Chromodynamics (QCD) is the self-coupling of the gluons due to their colour charges. The 'triple-gluon vertex' is a direct consequence of the non-Abelian nature of this gauge theory. The large two-jet rate for medium jet energies at hadron colliders can be considered as direct evidence for gluon-gluon scattering [1], if one accepts the extrapolation of the gluon structure function of the proton from deepinelastic $\nu N$ scattering to collider energies. A colourless gluon would lead to the reaction $\Upsilon \rightarrow 2$ jets [2], which is not observed [3]. In $e^{+} e^{-}$annihilation, the energy dependence [4] of the strong coupling constant $\alpha_{s}$, where the triple-gluon vertex enters through loop corrections, constitutes further indirect evidence for its existence. Direct evidence can be obtained from the study of 4-jet events in $e^{+} e^{-}$annihilation, since in four-parton final states the triple-gluon vertex contributes to the Born diagrams. Additional contributions to the 4-jet rate at Born-level originate from several classes of diagrams, e.g. those with double gluon bremsstrahlung and those in which a radiated gluon splits into a $q \bar{q}$ pair (secondary $q \bar{q}$ production) (Fig. 1 ).

In our previous analyses of 4 -jet events $[5,6]$, the two-dimensional distribution of the generalized Nachtmann-Reiter angle [7] versus the opening angle $\alpha_{34}$ of the two secondary jets was used. These two angles are defined in Fig. 2. The generalized Nachtmann-Reiter angle $\theta_{N R}^{*}$ distinguishes between the triple-gluon vertex contribution and secondary $q \bar{q}-$ production. The opening angle $\alpha_{34}$ distinguishes between the triple-gluon vertex and double-bremsstrahlung. Thus the triple-gluon vertex contribution may be determined directly from the two-dimensional event distribution in these two observables.

Our previous analyses used energy-ordering of the jets to distinguish between jets coming from primary and secondary partons. Other analyses of 4-jet events at LEP [8-10] have done the same. However, the primary quarks give the two highest energy jets in only $42 \%$ of the 4 -jet events. This leads to a smearing of the distributions of the observables for the different contributing diagrams, and diminishes the differences between them.

In this analysis, the primary jets were instead tagged by exploiting the long lifetime and the semileptonic decay of the primary hadrons from heavy quarks, which lead to detectable secondary vertices or to leptons with high transverse momenta. The higher purity of this tagging method results in larger differences between the shapes of the distributions of the observables for gluon bremsstrahlung, the triple-gluon vertex, and secondary quark production; it thus increases the sensitivity of the method.

\section{Theoretical Basis}

The triple-gluon vertex in $e^{+} e^{-}$annihilation appears in terms which are second order or higher in the strong coupling constant. The diagrams are shown in Fig. 1 for double bremsstrahlung, the triple-gluon vertex and secondary $q \bar{q}$ production. Thus testing the triple-gluon vertex requires a study of 4-jet events. For the rest of the paper, jets 1 and 2 correspond to the primary partons, jets 3 and 4 to the secondary partons.

The fundamental couplings are illustrated in Fig. 3. The Casimir factors $C_{F}, C_{A}$ and $T_{F}$ respectively measure the coupling strengths of gluon radiation from quarks, of the triple-gluon vertex and of gluon splitting into a quark-antiquark pair.

For any representation of a gauge group describing these couplings, the Casimir factors are determined in terms of its generators $t_{a b}^{r}$ and its structure constants $f^{r s t}$ by the relations (the notation of T. Hebbeker [11] is used): $t_{a b}^{r} t_{b c}^{r}=\delta_{a c} C_{F}, f^{r s t} f^{r s u}=\delta^{t u} C_{A}$, and 
$t_{a b}^{r} t_{b a}^{s}=\delta_{r s} T_{F}$, where $a, b, \ldots=1, \ldots, N_{C}$ is the quark color index, $r, s, \ldots=1, \ldots, N_{A}$ is the gluon color index, and repeated indices are to be summed. The ratio of the coupling strength $T_{F}$ for $g \rightarrow q \bar{q}$ to the coupling strength $C_{F}$ for $q \rightarrow q g$ is then given by [12]:

$$
T_{F} / C_{F}=N_{C} / N_{A}
$$

The interference terms contain combinations of these basic couplings and this leads to more complicated graphs for the transition probabilities. The graphs can be grouped into simple planar ones, and more complicated non-planar ones in which particle lines cross. Ellis, Ross and Terrano [14] have calculated the differential cross sections for the production of the four-parton final states to order $\alpha_{s}^{2}$. Their result is referred to below as the 'ERT' matrix element. Figs. 6 and 8 of their paper show all of the topologically distinct graphs for the transition probabilities. For the $q \bar{q} g g$ final state, there are 36 contributions which can be grouped into three classes:

A: planar double-bremsstrahlung graphs with weight $C_{F}^{2}$;

B: non-planar double-bremsstrahlung graphs with weight $C_{F}\left(C_{F}-\frac{1}{2} C_{A}\right)$;

$\mathrm{C}$ : graphs involving the triple-gluon vertex with weight $C_{F} C_{A}$.

Similarily the 36 contributions to the $q \bar{q} q \bar{q}$ final state fall into the following classes:

D: planar graphs with weight $C_{F} T_{R}$;

E: non-planar graphs with weight $C_{F}\left(C_{F}-\frac{1}{2} C_{A}\right)$;

$\mathrm{F}$ : graphs with weight $C_{F}$, which give contributions only if the charge of the partons is determined experimentally and are therefore not relevant to this analysis.

In these expressions, $T_{R}$ is given by:

$$
T_{R}=T_{F} n_{f}
$$

where $n_{f}=5$ is the number of active quark flavours. This number could be altered from its expectation of five by new physics, such as the existence of a very light gluino [13]. For greater generality, the original factor $N_{C}$ has been replaced by $C_{A}$, since this is the relevant coupling for the triple-gluon vertex [12]. In $\mathrm{SU}\left(N_{C}\right)$ gauge theory, and in particular in QCD, the quantities $C_{A}$ and $N_{C}$ are equal. In other gauge groups, however, the different physical meaning of these factors results in different numerical values.

The differential cross section for 4 -jet production in $e^{+} e^{-}$annihilation can be written in the form:

$$
\sigma_{4}\left(y_{i j}\right)=\sigma_{q \bar{q} g g}\left(y_{i j}\right)+\sigma_{q \bar{q} q \bar{q}}\left(y_{i j}\right) .
$$

Here $y_{i j} \equiv m_{i j}^{2} / s$ is the normalised effective mass squared for any pair of jets (partons).

The two contributions are therefore given by:

$$
\begin{aligned}
\sigma_{q \bar{q} g g}\left(y_{i j}\right) & =\sigma_{0} \alpha_{s}^{2} C_{F}^{2}\left[F_{A}\left(y_{i j}\right)+\left(1-\frac{1}{2} \frac{C_{A}}{C_{F}}\right) F_{B}\left(y_{i j}\right)+\frac{C_{A}}{C_{F}} F_{C}\left(y_{i j}\right)\right], \\
\sigma_{q \bar{q} q \bar{q}}\left(y_{i j}\right) & =\sigma_{0} \alpha_{s}^{2} C_{F}^{2}\left[\frac{T_{R}}{C_{F}} F_{D}\left(y_{i j}\right)+\left(1-\frac{1}{2} \frac{C_{A}}{C_{F}}\right) F_{E}\left(y_{i j}\right)\right],
\end{aligned}
$$

where $\sigma_{0}$ is the zeroth order 2 parton cross section and $F_{A}, \ldots, F_{E}$ are kinematic functions which correspond to the distributions for the classes A,...,E, for which the formulae are given in ref. [14]. These formulae are also used in the matrix element (ME) simulation in the JETSET [15] Monte Carlo Program, discussed further below.

Grouping the contributions with respect to the Casimir factors $C_{F}, C_{A}$ and $T_{R}$ gives:

$$
\sigma_{4}\left(y_{i j}\right)=\sigma_{0} \alpha_{s}^{2} C_{F}^{2}\left[F_{C F}\left(y_{i j}\right)+\frac{C_{A}}{C_{F}} F_{C A}\left(y_{i j}\right)+\frac{T_{R}}{C_{F}} F_{T R}\left(y_{i j}\right)\right]
$$




\begin{tabular}{|c|c|c||c|c|}
\hline Group & Gluons: & Quarks: & $N_{C} / N_{A}$ & $C_{A} / C_{F}$ \\
\hline \hline $\mathrm{SU}(n)$ & $n^{2}-1$ & $n$ & $\frac{n}{n^{2}-1}$ & $\frac{2 n^{2}}{n^{2}-1}$ \\
\hline$*$ & $n^{2}-1$ & $\frac{n(n-1)}{2}$ & $\frac{n}{2(n+1)}$ & $\frac{n^{2}}{(n+1)(n-2)}$ \\
\hline $\mathrm{SO}(n)$ & $\frac{n(n-1)}{2}$ & $n$ & $\frac{2}{n-1}$ & $2-N_{C} / N_{A}$ \\
\hline $\mathrm{Sp}(2 n)$ & $n(2 n+1)$ & $2 n$ & $\frac{2}{2 n+1}$ & $2+N_{C} / N_{A}$ \\
\hline$*$ & $n(2 n+1)$ & $2 n^{2}-n-1$ & $1-1 / n$ & $2-N_{C} / N_{A}$ \\
\hline$G_{2}$ & 14 & 7 & $1 / 2$ & 2 \\
\hline$F_{4}$ & 52 & 26 & $1 / 2$ & $3 / 2$ \\
\hline$E_{6}$ & 78 & 27 & $9 / 26$ & $18 / 13$ \\
\hline$E_{7}$ & 133 & 56 & $8 / 19$ & $24 / 19$ \\
\hline$E_{8}$ & 248 & 248 & 1 & 1 \\
\hline $\begin{array}{c}\mathrm{U}(1)_{3} \\
\text { Abelian }\end{array}$ & 1 & 3 & 3 & 0 \\
\hline $\begin{array}{c}\mathrm{U}(1) \\
\text { QED-like }\end{array}$ & 1 & 1 & 1 & 0 \\
\hline
\end{tabular}

Table 1: Expectations in different gauge theories for the ratio $N_{C} / N_{A}$ of the number of quark colours to the number of gluons, which is equal to the ratio $T_{F} / C_{F}$ between the $g \rightarrow q \bar{q}$ and $q \rightarrow q g$ coupling strengths, and for the ratio $C_{A} / C_{F}$ between the triple-gluon vertex and the $q \rightarrow q g$ coupling strengths. The quarks are assumed to be in the fundamental representation and the gluons in the adjoint representation, except for the lines marked with * where the quarks are in the next higher representation of the preceding gauge group.

where:

$$
\begin{aligned}
& F_{C F}\left(y_{i j}\right)=F_{A}\left(y_{i j}\right)+F_{B}\left(y_{i j}\right)+F_{E}\left(y_{i j}\right), \\
& F_{C A}\left(y_{i j}\right)=F_{C}\left(y_{i j}\right)-\frac{1}{2}\left(F_{B}\left(y_{i j}\right)+F_{E}\left(y_{i j}\right)\right), \\
& F_{T R}\left(y_{i j}\right)=F_{D}\left(y_{i j}\right) .
\end{aligned}
$$

For QCD, the Casimir factor giving the coupling strength of the $q \rightarrow q g$ vertex is $C_{F}=\frac{4}{3}$, that of the triple-gluon vertex is $C_{A}=3$, and that of the $g \rightarrow q \bar{q}$ vertex is $T_{F}=\frac{1}{2}$. For the Abelian model, the values are $C_{F}=1, C_{A}=0$, and $T_{F}=3$. For QED, the corresponding values are $C_{F}=1, C_{A}=0$, and $T_{F}=1$. The values for $N_{C} / N_{A}$ and $C_{A} / C_{F}$ in other gauge groups are given in Table 1 [12]. Since the dependence on the couplings, or Casimir operators, is gauge invariant, the ratios $C_{A} / C_{F}$ and $T_{R} / C_{F}$ can be determined by fitting the shape of the distribution. The results provide a test of QCD, and can also be compared with the predictions of other gauge groups.

In this analysis, integrated contributions of the classes are considered which are functions of only the two angular observables $\theta_{N R}^{*}$ and $\alpha_{34}$ defined previously (see Fig. 2). 
There is some correlation between $\theta_{N R}^{*}$ and $\alpha_{34}$; the study is therefore performed by fitting the two-dimensional distribution in these observables.

\section{Treatment of Data and $b$-quark tagging}

The analysis is based on 2.7 million multihadronic events from $e^{+} e^{-}$annihilations at centre of mass energies around the $Z^{0}$ resonance collected by the DELPHI detector in 1992, 1993 and 1994.

\subsection{The DELPHI Detector}

A detailed description of the DELPHI detector and its performance can be found elsewhere [16,17].

In the barrel region, the charged particle tracks are measured by a set of cylindrical tracking detectors whose axes are parallel to the $1.2 \mathrm{~T}$ solenoidal magnetic field and to the beam direction. The time projection chamber (TPC) is the main tracking device. The TPC is a cylinder with a length of $3 \mathrm{~m}$, an inner radius of $30 \mathrm{~cm}$ and an outer radius of 122 $\mathrm{cm}$. Between polar angles $\theta=39^{\circ}$ and $\theta=141^{\circ}$ with respect to the beam direction, tracks are reconstructed using up to 16 space points. The TPC also provides a measurement of the ionisation energy loss, $d E / d x$, in the drift gas. Additional precise $R \Phi$ measurements, in the plane perpendicular to the magnetic field, are provided at larger and smaller radii by the Outer and Inner detectors respectively. The Outer Detector (OD) has five layers of drift cells at radii between 198 and $206 \mathrm{~cm}$ and covers polar angles from $42^{\circ}$ to $138^{\circ}$. The Inner Detector (ID) is a cylindrical drift chamber having an inner radius of $12 \mathrm{~cm}$ and outer radius of $28 \mathrm{~cm}$. When the data used in this analysis were taken, it covered polar angles between $29^{\circ}$ to $151^{\circ}$ and contained a jet chamber section providing $24 R \Phi$ coordinates surrounded by five layers of proportional chambers providing both $R \Phi$ and longitudinal $z$ coordinates.

The vertex detector (VD) is located between the LEP beam pipe and the ID. It consists of three concentric layers of silicon microstrip detectors placed at radii of $6.3,9$ and $11 \mathrm{~cm}$ from the interaction region. For all layers, the microstrip detectors provide hits in the $R \Phi$ plane with a measured resolution including alignment errors of about $8 \mu \mathrm{m}$. For the data taken in 1994, the VD was upgraded so that the two layers at the smallest and largest radii also provided $z$ information.

In the forward and backward regions ( $\theta$ in the range $11^{\circ}-33^{\circ}$ or $\left.147^{\circ}-169^{\circ}\right)$ two additional drift chamber systems improve the tracking of charged particles. Forward chamber A (FCA) consists of three pairs of wire planes, rotated by $120^{\circ}$ with respect to each other in order to resolve internal ambiguities. Forward chamber B (FCB) consist of 12 wire planes, twice repeating the orientations of FCA.

The barrel electromagnetic calorimeter (HPC) covers the polar angles between $42^{\circ}$ and $138^{\circ}$. It is a gas-sampling device which provides complete three dimensional charge information in the same way as a time projection chamber. Each shower is sampled nine times in its longitudinal development. Along the drift direction, parallel to the DELPHI magnetic field, the shower is sampled every $3.5 \mathrm{~mm}$; in the plane perpendicular to the drift, the charge is collected by cathode pads of variable size ranging from $2.3 \mathrm{~cm}$ in the inner part of the detector to $7 \mathrm{~cm}$ in the outer layers. The excellent granularity allows good separation between close particles in three dimensions, and hence good electron identification even inside jets. The DELPHI Forward Electromagnetic Calorimeter (FEMC) subtends a polar angle $10^{\circ}<\theta<37^{\circ}$ and $143^{\circ}<\theta<170^{\circ}$. It consists of 
two $5 \mathrm{~m}$ diameter disks with a total of 9064 lead glass blocks in the form of truncated pyramids arranged to point just $3^{\circ}$ from the interaction point. The lead glass counters $\left(20 X_{0}\right.$ deep and $5 \times 5 \mathrm{~cm}^{2}$ in cross-section, i.e. $\left.\approx 1^{\circ} \times 1^{\circ}\right)$ are read out with vacuum photodiodes. The electron energy resolution is $\sigma / E=0.003 \oplus 0.12 / \sqrt{E} \oplus 0.11 / E$, with $E$ in $\mathrm{GeV}$, the last term being due to amplification noise.

Muon identification in the barrel region is based on a set of muon chambers (MUB), covering polar angles between $53^{\circ}$ and $127^{\circ}$. It consists of six active planes of drift chambers, two inside the return yoke of the magnet after $90 \mathrm{~cm}$ of iron (inner layer) and four outside after a further $20 \mathrm{~cm}$ of iron (outer and peripheral layers). The inner and outer modules have similar azimuthal coverages. The gaps in azimuth between adjacent modules are covered by the peripheral modules. Therefore a muon traverses typically either two inner layer chambers and two outer layer chambers, or just two peripheral layer chambers. Each chamber measures the $R \Phi$ coordinate to $2-3 \mathrm{~mm}$. Measuring $R \Phi$ in both the inner layer and the outer or peripheral layer determines the azimuthal angle of muon candidates leaving the return yoke within about $\pm 1^{\circ}$. These errors are much smaller than the effects of multiple scattering on muons traversing the iron. In the Forward part, the inner and outer layers each consist of two planes of drift chambers with anode wires crossed at right angles.

\subsection{Track and Event Selection}

Tracks were accepted only if their impact parameter relative to the nominal interaction vertex was below $5 \mathrm{~cm}$ in the plane transverse to the beam axis and below $10 \mathrm{~cm}$ along the beam direction, and their measured track length was above $50 \mathrm{~cm}$. Both charged and neutral particles were used in the event reconstruction. Photons were reconstructed as neutral showers in the HPC, or as photons converted in the material in front of the TPC. Particles were required to have momenta greater than $100 \mathrm{MeV} / \mathrm{c}$.

An event was accepted if the total visible energy was larger than $15 \mathrm{GeV}$ and each of the hemispheres $\cos \theta>0$ and $\cos \theta<0$ contained more than $3 \mathrm{GeV}$ visible energy, where $\theta$ is the angle to the beam direction. Hadronic decays, $Z^{0} \rightarrow q \bar{q}$, were selected by requiring at least 5 charged particles. Furthermore, for all events, the polar angle of the sphericity axis had to be between $40^{\circ}$ and $140^{\circ}$ and the total momentum imbalance below $20 \mathrm{GeV} / \mathrm{c}$. An event was excluded if a single photon carried more than $70 \%$ of the jet energy.

Only particles in the $25^{\circ}<\theta<155^{\circ}$ region were used. Jets were defined with the algorithm LUCLUS provided with JETSET. In this algorithm, two particles or jets with momenta $p_{1}$ and $p_{2}$ and opening angle $\alpha_{12}$ are merged if $2 \frac{p_{1} p_{2}}{p_{1}+p_{2}} \sin \frac{\alpha_{12}}{2} \leq d_{\text {join }}$. Each time two jets are merged, new jet axes are determined and all particles are reassigned to the nearest jet. With the new jets so defined, the procedure is repeated until a stable configuration is reached. In this analysis, the jet resolution parameter $d_{j o i n}$ was set to a fixed value of $4 \mathrm{GeV}$. This cut was optimized to suppress 4 jets coming from 3 parton events without losing to much signal. This selection yielded 398194 -jet events from the data of 1992/1993 and 41367 from 1994.

\subsection{Monte Carlo Simulation}

To determine the influence of detector effects on the analysis, JETSET matrix element (ME) events with full simulation of the DELPHI detector were used. The samples used for the 1992/1993 data comprised 30188 4-jet events from four parton final states and a 
separate production of 4058 4-jet events from 2-, 3- and 4-parton final states that was made in order to study the background to 4-jet events coming from initial states with less than 4 partons. For the 1994 data, a single sample of 70888 4-jet events coming from 2-, 3- and 4-parton final states was produced.

Consistency between the matrix element simulation and the real data was checked by comparing the thrust distributions of 4-jet samples and the particle transverse momenta in and out of the event plane with respect to the event axis, defined by the sphericity tensor. For each of jets 1 to 4, the distributions of the jet momenta, of the charged particle multiplicity in the jet, and of the transverse and longitudinal particle momenta relative to the jet axis were also examined. The average values and widths in data and simulation agreed typically within about $3 \%$.

\section{$3.4 \quad b$-tagging procedure}

This analysis requires a distinction between jets coming from the primary quarks and jets coming from gluons or secondary quarks. In practice, these separations are possible only for primary quarks with heavy flavours, using the large mass, long lifetime and distinctive decay modes of heavy-flavour hadrons. This separation is most effective for bottom quarks, which have the largest mass and longest lifetimes; furthermore, gluon splitting into bottom quarks is heavily suppressed. The methods for tagging $b$-quarks group into two basic categories: those using semileptonic decays and those using lifetime information.

The semileptonic tagging method consists of identifying high transverse momentum leptons in hadronic $Z^{0}$ decays. Muon identification [17] was based on an algorithm using a $\chi^{2}$ fit, using the degree of agreement between the extrapolated track and the track element constructed with the hits in the muon chambers. Electron identification [17] was performed using an algorithm combining the information from the HPC (based on a comparison of the deposited energy with the momentum measured by the tracking system, and on the longitudinal shape of the shower) and the $d E / d x$ measurement from the TPC. For the determination of the transverse momentum, the lepton was excluded from the jet and the new jet direction was calculated.

The lifetime method uses the fact that the finite lifetime of B-hadrons leads to decay products with large impact parameters. The distribution of impact parameters is characteristically larger in jets containing a B-hadron. A probability was calculated for the whole event, and also for each jet separately, that all the well-measured tracks belonging to the event or jet originated from the main vertex (compatible with the mean beam crossing point).

For this analysis, it was necessary to tag both $b$-jets in a 4 -jet event. It was therefore important to use an efficient tagging procedure. The lifetime-tag, lepton-tag and energy ordering methods were combined using a neural network. With respect to the use of the standard $b$-tagging procedure [17], which relies on lifetime information alone and is less efficient in the case of many jets, this increased the efficiency for tagging both $b$-jets in a 4 -jet event by over $50 \%$ for fixed purities of the tagged sample in the range $55 \%-85 \%$ (the purity used in the analysis was $70 \%$ ).

There were 13 input variables to the neural network: the transverse momenta of the electron in each jet, the transverse momenta of the muon in each jet, the lifetime probability for each jet, and the event lifetime probability. The network had one hidden layer and four output nodes, one for each jet. Output node one corresponded to the highest energy jet, and so on. Therefore energy ordering was also included. The output 
variables were normalized to be between 0 and 1 . A jet was tagged as a primary quark jet when the corresponding output variable had a value below 0.5 . An event was accepted if exactly two of the four jets were tagged as primary quark jets. This happened in $15.6 \pm 0.2 \%$ of all 4-jet events for Monte Carlo and in $15.9 \pm 0.2 \%$ for DELPHI data. In the accepted Monte Carlo event sample, the probability that both primary quarks were correctly identified was $70.0 \pm 0.7 \%$.

The net was trained to find $\mathrm{b}-q u a r k s$ with simulated ME events. In the training sample, the jet with the smallest angle with respect to the parton direction was taken as the corresponding jet. The data and the simulation were in good agreement, as is demonstrated in Fig. 4, which shows the output of the four nodes of the neural network.

Those events which passed the multihadron selection and were seen as a 4 jet event with two tagged jets were selected. This yielded 5794 data events and 4707 simulated events (4148 from the 4 parton ME, 559 from the 2-, 3- and 4-parton ME) for the 1992/1993 data, and 6795 data events and 12121 simulated events for 1994.

For the following analysis the jets selected as coming from primary quarks were treated as jets 1 and 2 .

\section{Analysis}

\subsection{Fit of the Casimir factors}

The 4-jet events from the data were binned into an $8 \times 8$ matrix according to their values of $\left|\cos \theta_{N R}^{*}\right|$ and $\cos \alpha_{34}$. The 1992/93 and 1994 data were considered separately because of the VD upgrade. The theoretical predictions $F_{C F}, F_{C A}$ and $F_{T R}$ were prepared as two-dimensional reference distributions $R_{C F}(l, m), R_{C A}(l, m)$ and $R_{T R}(l, m)$, in the form of $8 \times 8$ matrices in $\left|\cos \theta_{N R}^{*}\right|$ and $\cos \alpha_{34}$. These were produced from the JETSET 7.3 generator using the second order ERT matrix element [14] followed by a full simulation of the DELPHI detector. The theoretical prediction for the number of 4 -jet events is then given, for each bin $l, m$, by:

$$
T(l, m)=N\left[R_{C F}(l, m)+\frac{C_{A}}{C_{F}} \frac{R_{C A}(l, m)}{2.25}+\frac{T_{R}}{C_{F}} \frac{R_{T R}(l, m)}{1.875}\right],
$$

where $N$ is the overall normalisation factor. The denominators 2.25 and 1.875 take into account that the reference distributions were produced with the nominal QCD values of $C_{A} / C_{F}$ and $T_{R} / C_{F}$. The two-dimensional reference distributions and the distribution of DELPHI data are shown in Fig. 5.

The data are compared to:

$$
P(l, m)=T(l, m)+F(l, m),
$$

where $F(l, m)$ represents the background to the 4-jet events from fragmentation fluctuations of three and two parton events. Its contribution was determined from the full simulation of detector effects with the complete QCD matrix element and amounts to $0.96 \pm 0.09 \%$ of the 4 -jet events. The shape of this background differs from that of the reference distributions (see Fig. 5), so it is important to include it correctly into the fit.

A $\chi^{2}$ fit was then performed to the $\left|\cos \theta_{N R}^{*}\right|$ versus $\alpha_{34}$ distribution in terms of the two variables $X_{1}=\frac{C_{A}}{C_{F}}$, and $X_{2}=\frac{T_{R}}{C_{F}}$, using MINUIT [18] for minimisation. A combined fit to both variables allows the relative contribution of the three classes to be separated. 


\subsection{Details and Results of the Fitting Procedure}

The separate reference distributions $R_{C F}(l, m), R_{C A}(l, m)$ and $R_{T R}(l, m)$ cannot be generated directly on an event by event basis, since for some $\left(y_{i j}\right)$ configurations the contribution to $R_{C A}(l, m)$ becomes negative. The problem was solved as follows.

The $q \bar{q} g g$ and $q \bar{q} q \bar{q}$ events were generated in JETSET as usual, and followed through the detector simulation and tagging procedure. To calculate the reference distributions, for each $q \bar{q} g g$ event the weights $W_{A}, W_{B}, W_{C}$, and for each $q \bar{q} q \bar{q}$ event the weights $W_{D}$, $W_{E}$, as given in JETSET, were calculated (using $F_{A}, F_{B}, F_{C}$ and $F_{D}, F_{E}$ ) and stored. The weights in JETSET were defined as the contribution of each class to an event.

The reference distributions were then obtained by summing over the $q \bar{q} g g$ and $q \bar{q} q \bar{q}$ events in each bin:

$$
\begin{aligned}
R_{C F}(l, m) & =\sum_{i=1}^{N_{g g}} W_{C F}(i)+\sum_{i=1}^{N_{q \bar{q}}} W_{C E}(i) \\
R_{C A}(l, m) & =\sum_{i=1}^{N_{g g}} W_{C A}(i)-0.5 \sum_{i=1}^{N_{q \bar{q}}} W_{C E}(i) \\
R_{T R}(l, m) & =\sum_{i=1}^{N_{q \bar{q}}} W_{T R}(i)
\end{aligned}
$$

Here the following definitions have been used:

$$
\begin{aligned}
& W_{C F}(i)=C_{F}\left\{W_{A}(i)+W_{B}(i)\right\} / W_{g g}(i) \\
& W_{C A}(i)=C_{A}\left\{-0.5 W_{B}(i)+W_{C}(i)\right\} / W_{g g}(i) \\
& W_{T R}(i)=T_{R} W_{D}(i) / W_{q \bar{q}}(i) \\
& W_{C E}(i)=\left(C_{F}-0.5 C_{A}\right) W_{E}(i) / W_{q \bar{q}}(i)
\end{aligned}
$$

where $C_{F}=4 / 3$ and $C_{A}=3$ are the nominal $Q C D$ values and $W_{g g}$ and $W_{q \bar{q}}$ are the total weights for $q \bar{q} g g$ and $q \bar{q} q \bar{q}$ events respectively.

The squares of the weights for each event were also sorted into histograms. They allow calculation of the elements $v_{C F} v_{C F}, v_{C A} v_{C A}$ and $v_{C F} v_{C A}$ of the covariance matrix for $R_{C F}$ and $R_{C A}$ (class $\mathrm{E}$ contributes only about $0.3 \%$ to the events; its influence is neglected here):

$$
\begin{aligned}
v_{C F} v_{C F} & =\sum_{i=1}^{N_{g g}} W_{C F}^{2}(i) \\
v_{C A} v_{C A} & =\sum_{i=1}^{N_{g g}} W_{C A}^{2}(i) \\
v_{C F} v_{C A} & =0.5 \sum_{i=1}^{N_{g g}}\left(1-W_{C F}^{2}(i)-W_{C A}^{2}(i)\right) \text { since } W_{C F}(i)+W_{C A}(i)=1
\end{aligned}
$$

The reference distributions $R_{C F}$ and $R_{C A}$ have some correlations, since they originate from the same events. Using the covariance matrix, this can be taken into account correctly. Note that the $q \bar{q} q \bar{q}$ events originate from class D only, if class $\mathrm{E}$ is neglected, so $R_{T R}$ is not correlated with $R_{C A}$ and $R_{C F}$.

The background to 4-jet events coming from fragmentation fluctuations of 3 - and 2parton events (Fig. 5) was included, together with the propagation of its statistical error. The influence of the background is therefore included in the total statistical error. 
In the standard fits, only 55 bins of the $8 \times 8$ matrix were used. The 8 bins in the row near $\cos \alpha_{34}=1$ were omitted, together with the bin nearest $\left|\cos \theta_{N R}^{*}\right|=1, \cos \alpha_{34}=-1$. These bins contain only a few events which, in addition, are at the boundaries of the two-jet resolution and are therefore less reliable in the simulation. The fit gives a $\chi^{2}$ of 56.4 at 53 d.o.f.

The combined fit results from the $1992 / 93$ and 1994 data were

$$
\begin{aligned}
& C_{A} / C_{F}=2.51 \pm 0.25 \text { (stat.) } \\
& T_{R} / C_{F}=1.91 \pm 0.44 \text { (stat.) }
\end{aligned}
$$

where $T_{R}=n_{f} T_{F}$.

The production of secondary heavy quark-antiquark pairs is kinematically suppressed. The quark tagging algorithm increases the ratio of secondary heavy flavours again. A separate study with the applied jet cut has shown that the different flavours are selected in the ratio $d: u: s: c: b=1: 1:(1.11 \pm 0.06):(1.50 \pm 0.07):(1.95 \pm 0.07)$. This enrichment in heavy quarks due to the tagging algorithm is already built into the reference distributions which are used in the fit. Since the number of secondary quarks is known to be 5 , one has to divide by 5 to reduce the result for $T_{R} / C_{F}$ to the value $T_{F} / C_{F}$ for one quark flavour.

\section{$5 \quad$ Systematic Errors}

\subsection{Fragmentation}

To study the influence of the variation of the fragmentation parameters on the results, the five fragmentation parameters [15], namely $a$ and $b$ for the LUND fragmentation function, $\epsilon_{c}$ and $\epsilon_{b}$ for the Peterson fragmentation function, and $\sigma_{q}$, were chosen independently in large ranges around their nominal values (see table 5.1), using a random generator. The fragmentation cut-off and QCD scale parameters were varied similarly, and Bose-Einstein correlations were switched off and on.

\begin{tabular}{|c|c||c|c|}
\hline Parameter & $\begin{array}{c}\text { Name inside } \\
\text { Jetset 7.3 }\end{array}$ & Value & Variation $\sigma$ \\
\hline \hline Lund $a$ & PARJ(41) & 0.9 & \pm 0.18 \\
\hline Lund $b$ & PARJ(42) & 0.534 & \pm 0.10 \\
\hline Peterson $\epsilon_{c}$ & PARJ(54) & -0.075 & \pm 0.014 \\
\hline Peterson $\epsilon_{b}$ & PARJ(55) & -0.008 & \pm 0.002 \\
\hline Transverse Momentum $\sigma_{q}$ & PARJ(21) & 0.415 & \pm 0.08 \\
\hline Fragmentation Cut off & PARJ(35) & 1.9 & \pm 0.38 \\
\hline QCD Scale & PARJ(122) & 0.17 & \pm 0.034 \\
\hline Bose-Einstein Correlation & MSTJ(51) & 0 or 1 & - \\
\hline
\end{tabular}

Table 2: Parameter variations used in evaluation of fragmentation systematic. 
The same large sample of 4-parton events was used to study the fragmentation for each set of parameters. A simple $b$-tagging algorithm and a simple detector simulation were included at generator level. The fragmentation was done for 500 different sets of parameters. The resulting sets of events were then considered as data. The values of $C_{A} / C_{F}$ and $T_{R} / C_{F}$ for these data sets were then compared with those from 500 sets generated with the nominal fragmentation parameters. The root-mean-square shifts in $C_{A} / C_{F}$ and $T_{R} / C_{F}$ were \pm 0.11 and \pm 0.25 , respectively.

\subsection{Influence of higher orders}

No complete $O\left(\alpha_{s}^{3}\right)$ calculations of the jet cross sections exist. Therefore it is not possible to determine the influence of higher orders exactly. In order to get an estimate, the 5 -parton Born cross section [19] has been implemented in the JETSET event generator [20]. With our cuts, about $37 \%$ of the 5 -parton events are seen as 4 -jet events. Experimentally the ratio of 5-jet to 4 -jet events is about $5.6 \%$. The observed 5-jet rate has contributions from all higher orders. From a migration efficiency of $37 \%$ from 5 -partons to 4 -jet events, the background from higher orders is estimated to be about $3 \%$ in the 4-jet sample, so the background is small. Since, in addition, the distributions in $\theta_{N R}^{*}$ and $\alpha_{34}$ for 5 -parton events were found to be similar to those for 4-parton events, the influence of the 5-parton background on the measurement of $C_{A} / C_{F}$ and $T_{R} / C_{F}$ was neglected.

\subsection{Influence of heavy quark masses}

The ERT matrix element includes the effects of quark masses only on the parton energies, but not their effects on the angular structure of the event. The OPAL collaboration investigated the effect with generators including this mass effect fully [10]. It was found not to influence the result on $C_{A} / C_{F}$ and to have only a small effect on $T_{R} / C_{F}$.

\subsection{Dependence on $y_{\text {cut }}^{p}$}

In the calculation of the parton cross-sections, a $y_{c u t}^{p}$ is applied at the parton level to handle the divergences from soft and collinear gluons, with $y_{i j}^{p}=2 E_{i} E_{j}\left(1-\cos \theta_{i j}\right) / E^{2}$ and $E=\Sigma_{i} E i$. On the parton level, $y_{c u t}^{p}=0.01$ in the generator is below the cut imposed on the kinematic configuration by the value of $d_{\text {join }}$ applied on the hadron level in the LUCLUS cluster routine. When lowering $y_{c u t}^{p}$, additional softer partons are produced, but after applying the cluster routine with the value of $d_{\text {join }}$ used at the hadron level to the parton configuration, exactly the same partons survive. This independence of the $y_{c u t}^{p}$ value is not perfect for the jets reconstructed from the hadrons, due to fluctuations in the fragmentation of the partons.

The influence of the change in the $y_{c u t}^{p}$ on the result of the analysis was determined by generating 50 samples of events with $y_{\text {cut }}^{p}=0.011,0.012$ and 0.014 . This was done using the same simple $b$-tagging algorithm as for the fragmentation study. The events were fitted against the real data. There was no significant shift with respect to the result using the value $y_{\text {cut }}^{p}=0.010$. 


\subsection{Dependence on the $b$-tagging procedure}

The procedure was used with different calibrations of the VD and its errors and with different network designs. The result of the fit of the Casimir factors did not change noticeably.

The shapes of the reference distributions are different from each other also for jets which are assigned incorrectly (see Fig. 6). To estimate the influence of the b-tagging procedure, two types of reference distributions (correctly tagged and incorrectly tagged) were generated and added with different weights to get reference distributions with different purities. For purity values in the range $60 \%-80 \%$, the values of $C_{A} / C_{F}$ and $T_{R} / C_{F}$ obtained by fitting these distributions to the DELPHI data were found to vary linearly with purity. Assuming an uncertainty of $\pm 3 \%$ for the purity gave systematic errors of $\mp 0.07$ for $C_{A} / C_{F}$ and \pm 0.03 for $T_{R} / C_{F}$.

\subsection{Dependence on the cluster algorithm}

In the analysis, jets are defined with the LUCLUS algorithm. To investigate the influence of the cluster algorithm, the analysis was repeated using the JADE algorithm [15] with $y_{c u t}=0.02$ and the jet resolution parameters were varied by $\pm 20 \%$ for both algorithms. No systematic effects were observed, so no additional systematic error was included.

\section{Discussion and Conclusions}

The combined results of the analysis of the $1992 / 93$ and 1994 data are:

$$
C_{A} / C_{F}=2.51 \pm 0.25 \text { (stat.) } \pm 0.11 \text { (fragm.) } \pm 0.07 \text { (b-tagging) }
$$

and

$$
T_{R} / C_{F}=1.91 \pm 0.44 \text { (stat.) } \pm 0.25 \text { (fragm.) } \pm 0.03 \text { (b-tagging) }
$$

where $T_{R}=n_{f} T_{F}$. The possible influence of higher order terms provides an additional unquantifiable uncertainty which, in the following discussion, is assumed to be negligible.

Since $T_{F} / C_{F}=N_{C} / N_{A}$ and $n_{f}=5$, one can obtain the ratio for the number of quark colours $N_{C}$ to the number of gluon colours $N_{A}$. Adding the errors in quadrature results in

$$
C_{A} / C_{F}=2.51 \pm 0.30 \quad \text { and } \quad N_{C} / N_{A}=0.38 \pm 0.10 .
$$

The result for $C_{A} / C_{F}$ is in agreement with the value $9 / 4$ expected for $Q C D$. The value for $N_{C} / N_{A}$ is consistent with the QCD value of $3 / 8$. The errors are substantially smaller than in our previously published result, $C_{A} / C_{F}=2.12 \pm 0.35$ and $N_{C} / N_{A}=0.46 \pm 0.19[6]$, obtained without $b$-tagging. The $68 \%$ and $95 \%$ confidence level contours are given in Fig. 7 .

At leading order, a massless gluino would lead to an excess in the number of active flavours. Mass effects can lower this excess. The light gluino hypothesis is not favoured by these measurements, but a full discussion of this point would require detailed simulation of the possible effects on the analysis of any undetected gluino decay products and of the long lifetime of very light gluinos. In addition the fragmentation of the gluino is not known and the effect of the b-tagging procedure is therefore unclear. We therefore do not consider this result to be strong evidence against the light gluino hypothesis. 
The measured variables $C_{A} / C_{F}$ and $N_{C} / N_{A}$ represent the ratios of the coupling strength of the triple-gluon vertex to that of gluon bremsstrahlung from a quark, and of the number of quark colours to the number of gluons. The result shows that the triple-

gluon vertex must exist and that the number of quark colours has to be smaller than the number of gluons.

The expectations for various other gauge groups are also given in Table 1 and Fig. 7 . The quarks are assumed to be in the fundamental representation and the gluons in the adjoint representation, except for $\mathrm{SU}(4)^{\prime}, \mathrm{SP}(4)^{\prime}$, and $\mathrm{SP}(6)^{\prime}$, which are examples with quarks in the next higher representation.

From Table 1, it is evident that most groups in the plot are excluded already by their number of quark colours, which is also restricted experimentally to $N_{C}=3$ by the hadronic cross section in $e^{+} e^{-}$annihilation, usually expressed as $R=\sigma\left(e^{+} e^{-} \rightarrow\right.$ hadrons $) / \sigma\left(e^{+} e^{-} \rightarrow \mu^{+} \mu^{-}\right)$, and by the decay width of the $\pi^{0}$ into two photons via quark loops.

Apart from SU(3) and the Abelian $\mathrm{U}(1)_{3}$ model, which was invented ad hoc, only $\mathrm{SO}(3)$ has 3 colours for the quarks. But it has only 3 gluons, in contrast to the 8 gluons in QCD. Our result excludes $\mathrm{SO}(3)$ (and $\mathrm{U}(1)_{3}$ ) as candidates and establishes that $8 \pm 2$ gluons exist in nature.

\section{Acknowledgements}

Our thanks go to K. Chetyrkin, R. Decker, and T. Sjöstrand for many helpful discussions. The physical interpretation of the results in the context of gauge groups was clarified by contributions from M. Jezabek. We are greatly indebted to our technical collaborators and to the funding agencies for their support in building and operating the DELPHI detector, and to the members of the CERN-SL Division for the excellent performance of the LEP collider. 


\section{References}

[1] L. DiLella, Ann. Rev. Nucl. Part. Sci. 35 (1985) 107.

[2] T.F. Walsh and P.M. Zerwas, Phys. Lett. B93 (1980) 53.

[3] ARGUS Coll., H. Albrecht et al., Z. Physik C31 (1986) 181.

[4] S. Bethke: Plenary talk at the Dallas 1992 Conference.

[5] DELPHI Coll., P. Abreu et al., Phys. Lett. B255 (1991) 466.

[6] DELPHI Coll., P. Abreu et al., Z. Physik C59 (1993) 357.

[7] G. Rudolph, Physics at LEP, CERN 86-02 (1986) Vol. 2, 150.

[8] ALEPH Coll., R. Barate et al., CERN-PPE/97-002 (1997).

[9] L3 Coll., B. Adeva et al., Phys. Lett. B248 (1990) 227.

[10] OPAL Coll., R. Akers et al., Zeit. Phys. C65 (1995) 367.

[11] T. Hebbeker, Phys. Rep. 217 (1992) 69.

[12] M. Jezabek, private communications.

[13] G. Farrar, Phys. Rev. D51 (1995) 3904.

[14] R. K Ellis, D. A. Ross and A. E. Terrano, Nucl. Phys. B178 (1981) 421.

[15] T. Sjöstrand, Comp. Phys. Comm. 28 (1983) 229;

T. Sjöstrand, "PYTHIA 5.6 and JETSET 7.3", CERN-TH.6488/92 (1992).

[16] DELPHI Coll., P. Aarnio et al., Nucl. Instr. Meth. A303 (1991) 233.

[17] DELPHI Coll., P. Abreu et al., Nucl. Instr. Meth. A378 (1996) 57.

[18] F. James and M. Roos, CERN library program D506 on GENLIB.

[19] F. A. Berends, W. T. Giele and H. Kuijf, Nucl. Phys. B321 (1989) 39.

[20] Frank Wäckerle, Diploma thesis, Univ. Karlsruhe IEKP-KA/93-19 (1993). 

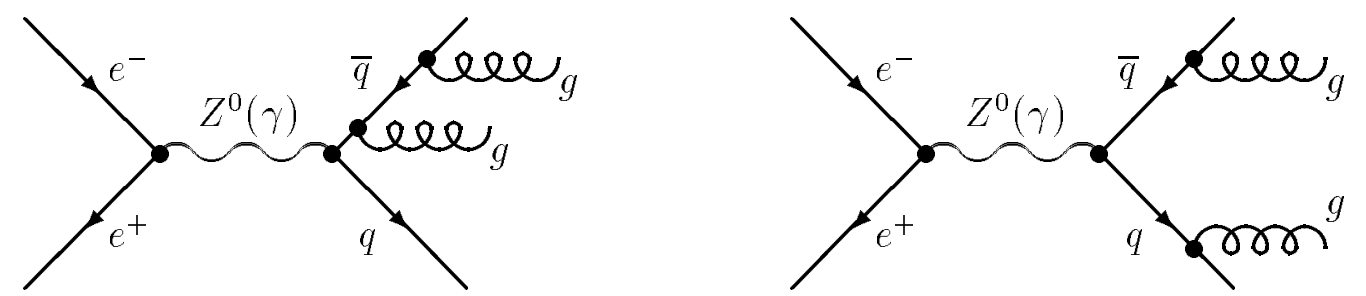

(a)

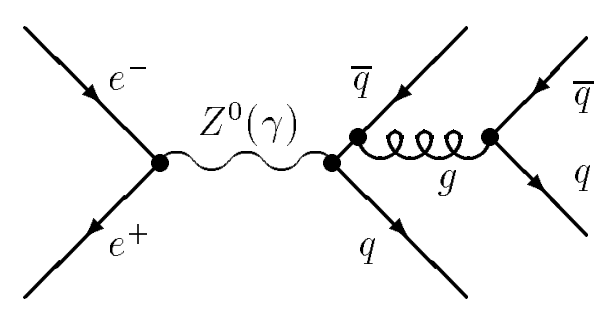

(b)

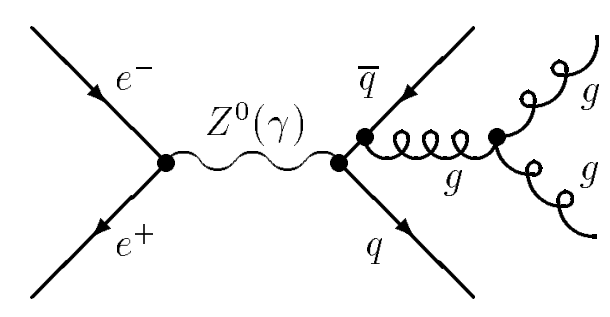

(c)

Figure 1: Diagrams yielding four-parton final states: (a) double gluon bremsstrahlung, (b) secondary $q \bar{q}$ pair production, (c) triple-gluon vertex.

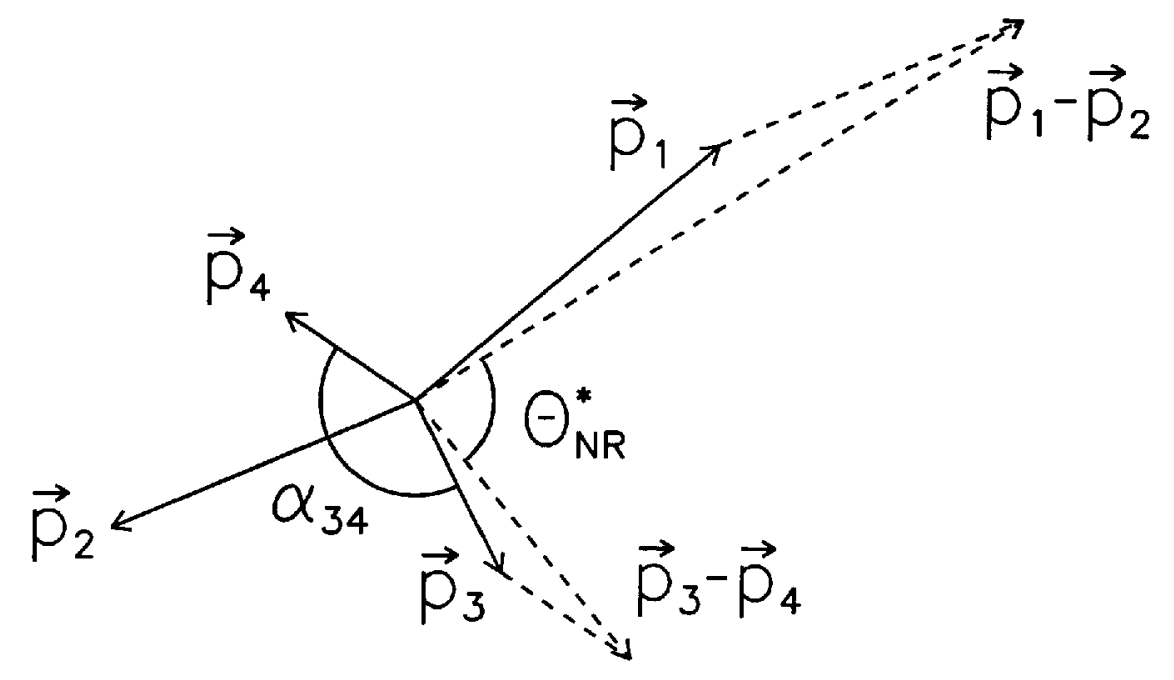

Figure 2: Definitions of the generalised Nachtmann-Reiter angle $\theta_{N R}^{*}$ and of the angle $\alpha_{34}$, which are the two angles used in the analysis: $\vec{p}_{1}$ and $\vec{p}_{2}$ are the momentum vectors of the two primary jets, $\vec{p}_{3}$ and $\vec{p}_{4}$ are those of the secondary jets, $\theta_{N R}^{*}$ is the angle between $\vec{p}_{1}-\vec{p}_{2}$ and $\vec{p}_{3}-\vec{p}_{4}$, while $\alpha_{34}$ is the angle between $\vec{p}_{3}$ and $\vec{p}_{4}$. 
(a)

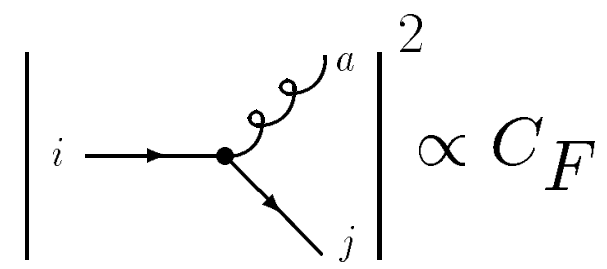

(b)

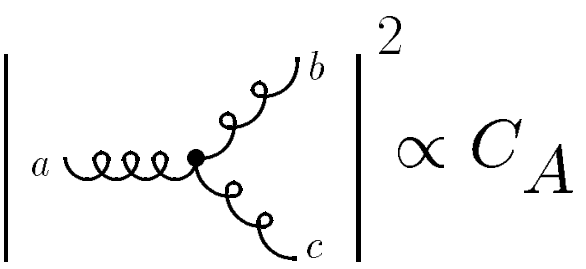

(c)

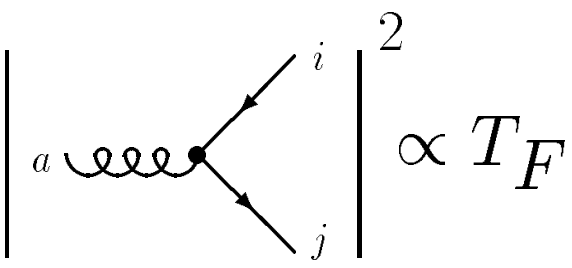

Figure 3: Casimir factors for the fundamental couplings. Diagrams (a) and (c) have the same $q \bar{q} g$ topology; their relative coupling strengths are determined by statistical factors, i.e. they are related to the numbers of quark colours $N_{C}$ and the number of gluons $N_{A}$ by $T_{F} / C_{F}=N_{C} / N_{A}$. 

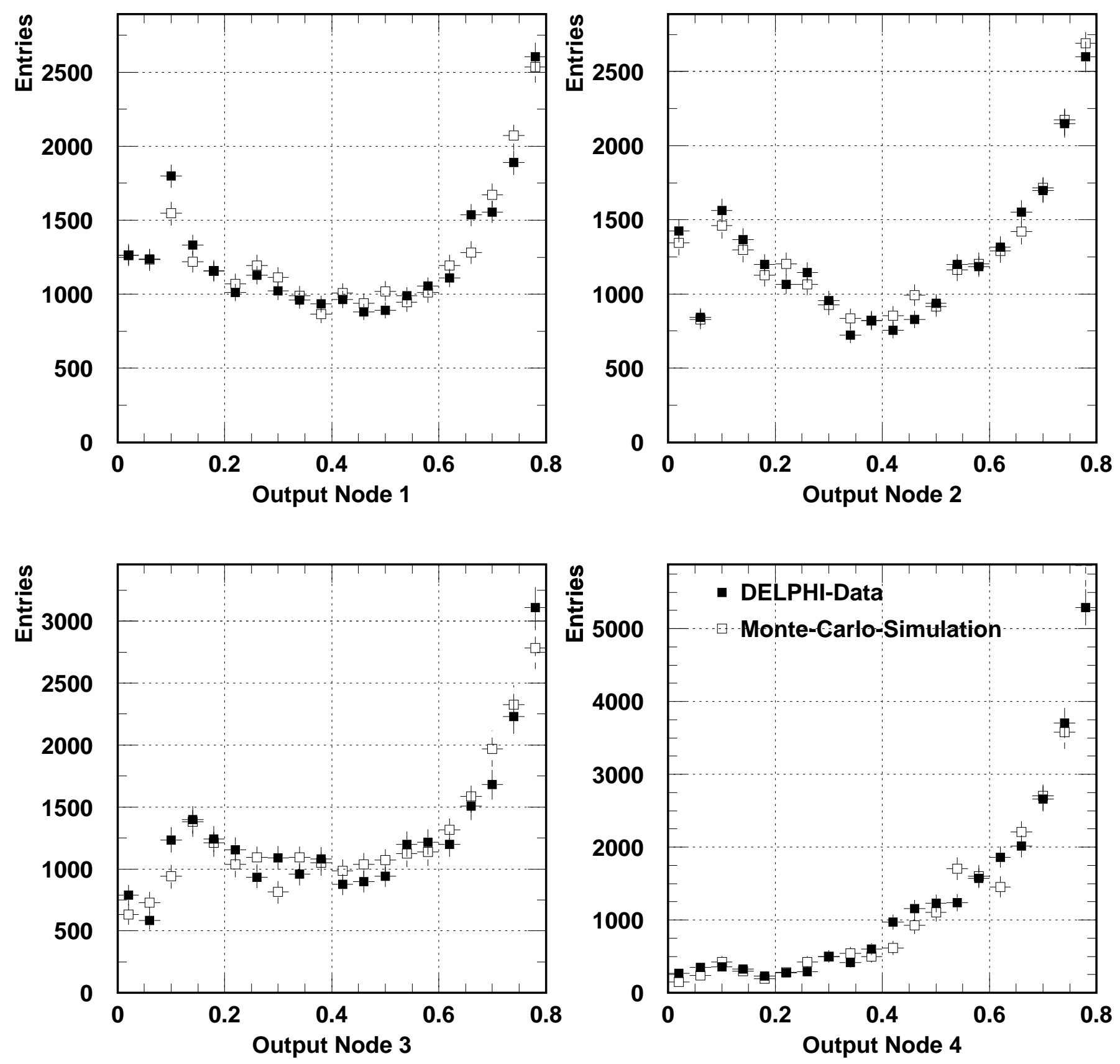

Figure 4: Output of the four different nodes of the neural network using DELPHI data (filled boxes) and simulation (open boxes). Nodes 1-4 correspond to jets 1-4 numbered in order of decreasing energy. All of the distributions have high peaks for outputs above 0.8 (not shown). A jet with output below 0.5 was classified as a quark jet. Only events with exactly two jets classed as quark jets were used in the analysis. 


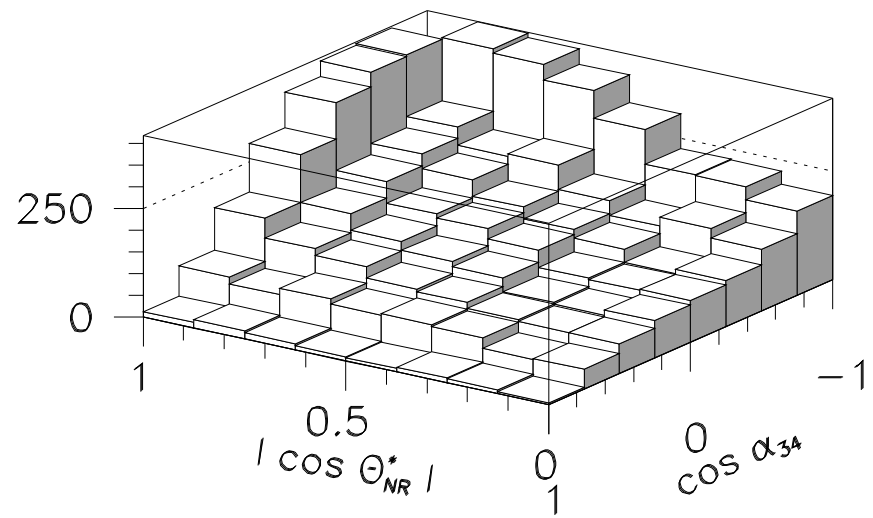

\section{reference distribution $R_{C F}$}

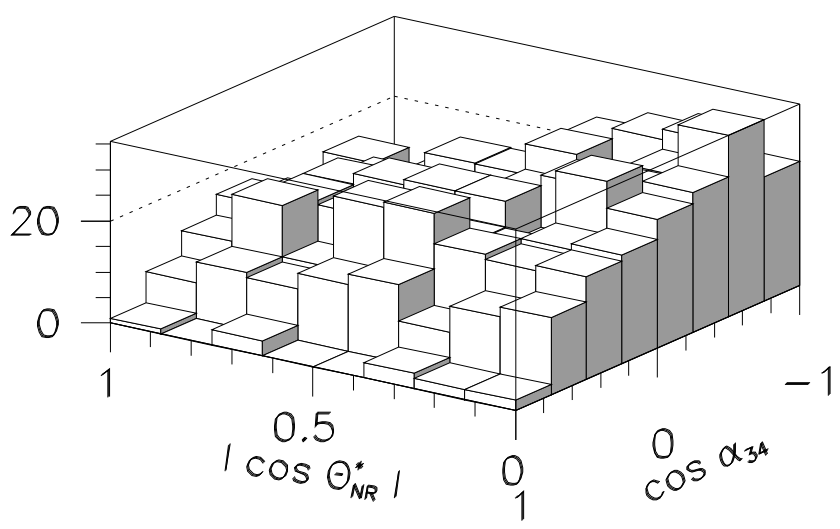

reference distribution $R_{T R}$

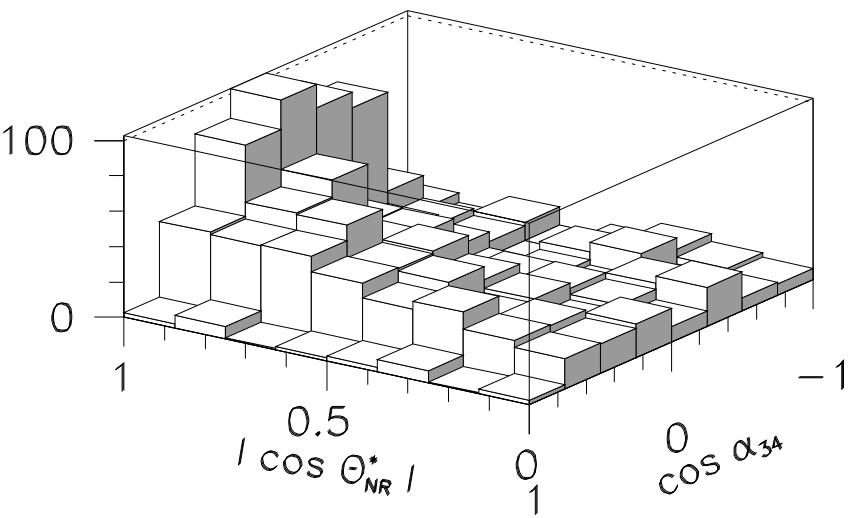

reference distribution $\mathbf{R}_{\text {CA }}$

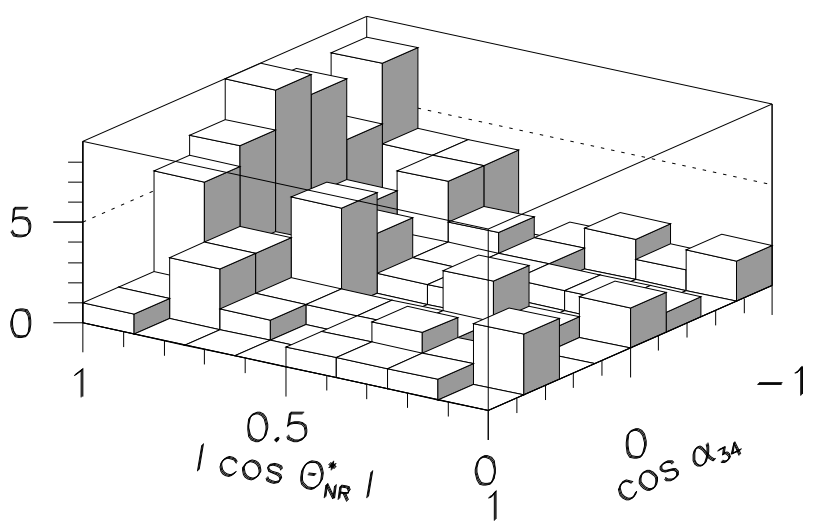

background

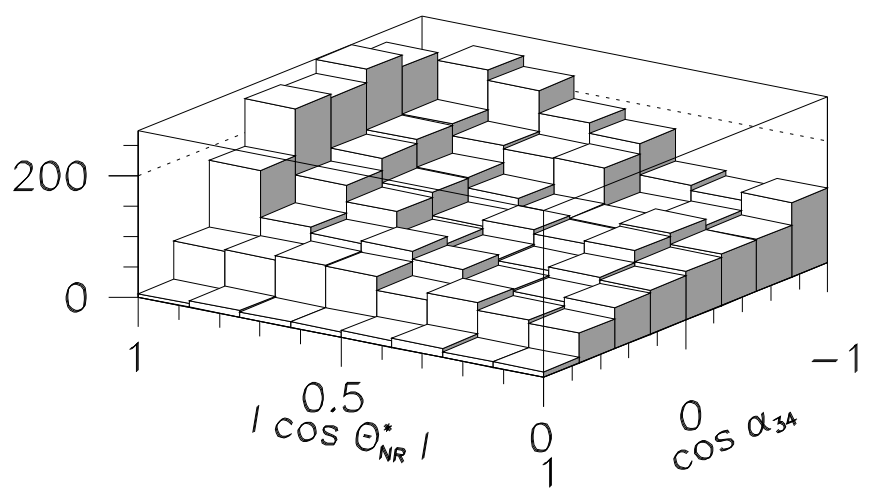

DELPHI-Data

Figure 5: Two-dimensional distributions in $\left|\cos \theta_{N R}^{*}\right|$ and $\cos \alpha_{34}$. The three reference distributions $R_{C F}$ (double gluon bremsstrahlung), $R_{C A}$ (triple gluon vertex), and $R_{T R}$ (secondary $q \bar{q}$ pair production), normalized according to formula 10 , and the distribution of the background of 3-parton events seen as 4 jets, are fitted to the DELPHI data distribution. The generalized Nachtmann-Reiter angle $\theta_{N R}^{*}$ distinguishes $R_{C A}$ from $R_{T R}$, The opening angle $\alpha_{34}$ distinguishes $R_{C A}$ from $R_{C F}$. 

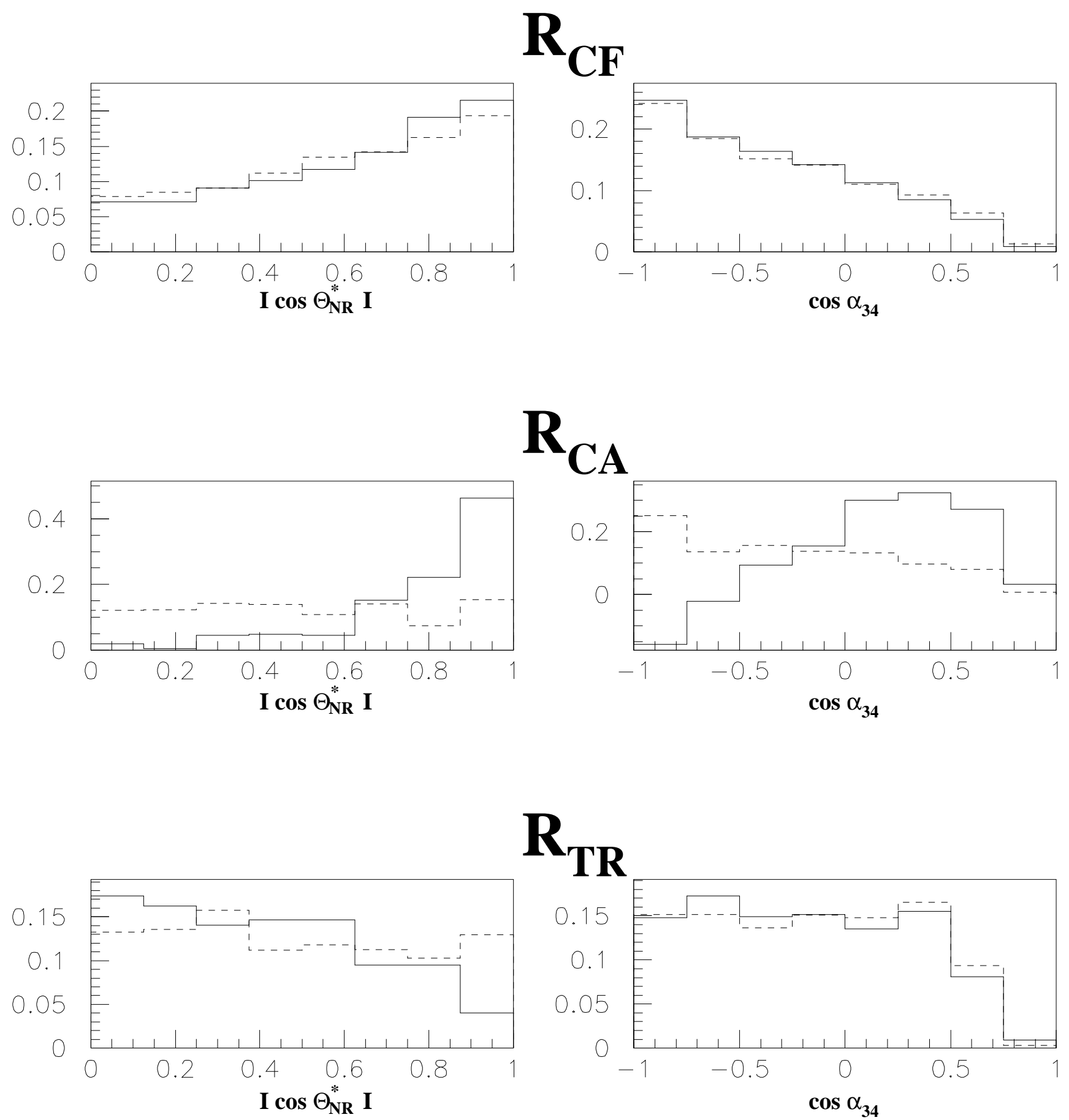

Figure 6: Comparison of the shapes of the projections on the $\left|\cos \theta_{N R}^{*}\right|$ and $\cos \alpha_{34}$ axes of the two-dimensional distributions used in the fit. The full lines show the shapes of the distributions of correctly $b$-tagged events, the dashed lines show those of the events in which the jets are assigned incorrectly. 


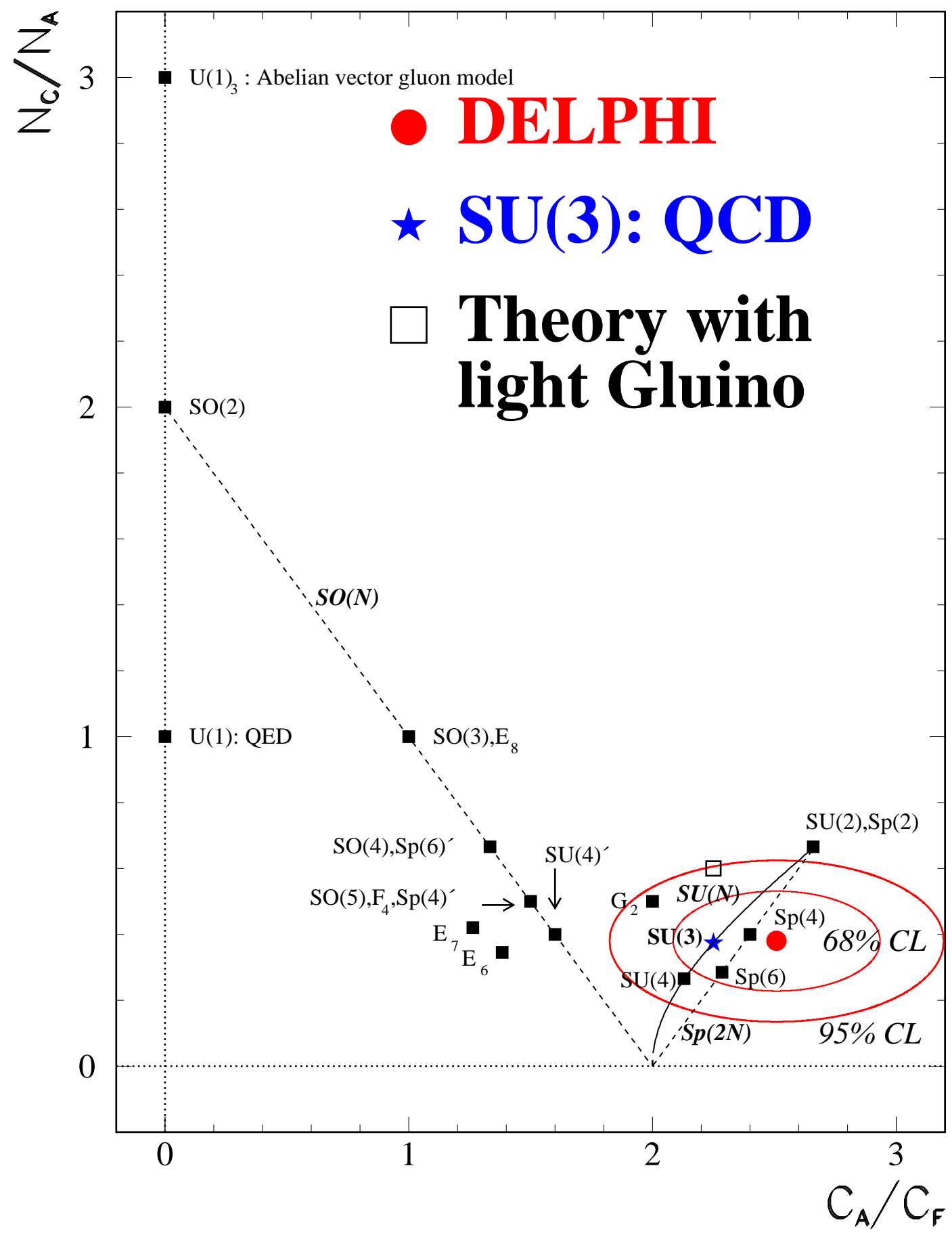

Figure 7: $68 \%$ and $95 \% \mathrm{CL}$ contour plots for the measured variables $C_{A} / C_{F}$ and $N_{C} / N_{A}$, and expectations from different gauge theories including QCD with and without a light gluino. $C_{A} / C_{F}$ is the coupling strength ratio of $g \rightarrow g g$ to $q \rightarrow q g$, and $N_{C} / N_{A}=T_{F} / C_{F}$ is the number of quark colours divided by the number of gluons. The results clearly show that the triple-gluon vertex must exist and that the number of gluons is larger than the number of quark colours. 